\title{
Aspectos clínicos e diagnósticos da leishmaniose tegumentar americana em militares simultaneamente expostos à infecção na Amazônia
}

\author{
Clinical and diagnostic aspects of American tegumentary leishmaniosis in \\ soldiers simultaneously exposed to the infection in the Amazon Region
}

\author{
Jorge Augusto de Oliveira Guerra ${ }^{1}$, Sinésio Talhari, ${ }^{1,2}$ Marcilene Gomes Paes ${ }^{1}$ \\ Marlúcia Garrido ${ }^{1}$ e Joana Maria Talhari'
}

\begin{abstract}
Resumo Os treinamentos militares na selva amazônica constituem importantes fatores na incidência de leishmaniose tegumentar americana na região. Este trabalho descreve 48 casos de leishmaniose tegumentar americana em pacientes militares nos quais a doença se manifestou após operação de treinamento na Amazônia. O período de incubação médio foi de 27,6 dias. Houve predomínio de lesões nos membros superiores, face e pescoço. A maioria dos indivíduos apresentou duas lesões, sendo as úlceras as manifestações mais freqüentes. O diagnóstico foi realizado pelo exame de escarificação das lesões, sendo confirmado pela demonstração do parasito em $43(89,6 \%)$ casos.
\end{abstract}

Palavras-chaves: Leishmaniose. Militares. Treinamento. Selva amazônica.

Abstract Military exercises in the forest of the Amazon region constitute an important factor in the incidence of American cutaneous leishmaniasis in this region. This report describes 48 cases of American tegumentary leishmaniosis. All these patients acquired the disease after military exercises in the Amazon. The mean incubation time was 27.6 days. The lesions were predominantly in the upper extremities, face and neck. The majority of patients presented two lesions. Ulcers were the most common clinical manifestation. The diagnosis was obtained through skin smears of the lesions. A total of 43 cases (89.6\%) were found to be positive.

Key-words: Leishmaniasis. Military. Exercises. Amazon forest.

Na Amazônia, e em particular no Estado do Amazonas, a incidência da leishmaniose tegumentar americana (LTA) tem se mantido elevada. A abertura de novas estradas, a instalação de núcleos residenciais em áreas de floresta e os treinamentos militares constituem fatores importantes na epidemiologia da leishmaniose ${ }^{11} 17$. Os treinamentos militares são freqüentes na Amazônia e a ocorrência de casos na tropa é alta.

No Estado do Amazonas, no período de 1976 a 1980, foram diagnosticados 2.006 casos de LTA, a maioria em habitantes das margens das estradas e da periferia de Manaus ${ }^{5}$. De 1985 a 2000, foram registrados 30.251 casos de LTA, sendo 17.374 $(57,4 \%)$ procedentes do município de Manaus ${ }^{11}$.
A Fundação de Medicina Tropical (FMT/IMT-AM), que atende em média $47,5 \%$ dos casos de leishmaniose do Estado, vem registrando em torno de 1.000 casos novos a cada ano, quase todos oriundos do município de Manaus, principalmente de duas estradas: AM-010, que liga Manaus ao município de Itacoatiara e BR-174, que liga Manaus a Boa Vista, capital do Estado de Roraima.

No município de Manaus, há nítido predomínio da LTA causada pela Leishmania (Viannia) guyanensis, cujo principal transmissor é a Lutzomyia umbratilis, sendo a Lu. anduzei, um vetor secundário 2101315 . A Leishmania (Leishmania) amazonensis é um outro agente de LTA na região, cujos transmissores são a $L u$. flavíscutellata e Lu. Olmeca nociva ${ }^{15}$, acreditando-se

1.Fundação de Medicina Tropical do Amazonas, Manaus, AM. 2.Universidade do Amazonas, Manaus, AM.

Endereço para correspondência: Dr. Jorge Augusto de Oliveira Guerra. Gerência de Leishmanioses/FMT-AM. Av. Pedro Teixeira 25, D. Pedro I, 69040-000 Manaus, AM.

Fax: 5592 238-3762

e-mail: jguerra@horizon.com.br

Recebido para publicação em 8/4/2002

Aceito em 23/4/2003 
que o rio Amazonas constitua barreira geográfica para as outras espécies agentes de LTA na região ${ }^{12} 15$.

Do ponto de vista clínico, o aspecto das lesões é semelhante, independentemente das espécies de Leishmania identificadas. Entretanto, em nosso meio, observam-se algumas características que podem diferenciá-las daquelas cujo agente etiológico é a
Leishmania (Viannia) braziliensis, a qual predomina ao sul da calha do rio Amazonas, distribuindo-se nos estados das demais regiões do país ${ }^{10}{ }^{12}$ 13, observase, na Leishmania (Viannia) guyanensis, maior freqüência de linfonodos adjacentes às lesões, menor tendência para metástase nasal ${ }^{516}$ e a necessidade de maior tempo de tratamento.

\section{PACIENTES E MÉTODOS}

São descritos 48 casos de LTA, atendidos na FMT de janeiro a março de 1995. Todos os pacientes pertenciam ao contigente militar do 7ํㅡㄴ Comando Aéreo de Manaus (70 COMAR). Um grupo de 98 pessoas participou de uma operação em uma base de treinamento (Base Boina Verde) localizada no quilometro 23 da Rodovia AM-010 (Manaus-Itacoatiara), no período de 16 a 23 de novembro de 1994.

Para a confirmação do diagnóstico clínico de LTA foram realizados: pesquisa direta da Leishmania nas lesões, sendo o material corado pelo GIEMSA, naqueles que tiveram a pesquisa direta negativa foram realizados Intradermorreação de Montenegro (IDRM) e exame histopatológico onde parte do material também foi semeado em meio de cultura.

Todos os doentes foram tratados com N-metilglucamina ou com pentamidina conforme recomendação do Ministério da Saúde ${ }^{9}$. Os antimoniais foram utilizados na dose de $15 \mathrm{mg} / \mathrm{kg} /$ dia por 20 dias, correspondendo a uma série de tratamento. Nos casos de não fechamento das lesões, utilizou-se uma nova série de tratamento por mais 20 dias. Nos pacientes que não responderam à terapêutica com duas séries de antimoniais, ou naqueles com efeitos colaterais mais intensos, utilizou-se pentamidina como segunda linha de tratamento.

A pentamidina foi utilizada na dose de $4 \mathrm{mg} / \mathrm{kg} / \mathrm{dose}$, em três aplicações intramusculares, com intervalo de dois dias entre cada aplicação, correspondendo a uma série de tratamento. Quando persistiu atividade nas lesões após um prazo de observação de 60 dias, utilizou-se nova série da medicação.

Consideram-se curados aqueles pacientes que se encontravam no seguimento após uso da medicação com suas lesões em cicatrização e que não retornaram ao serviço.

\section{RESULTADOS}

Os 48 pacientes eram do sexo masculino. A faixa etária mais acometida foi de 19 e 20 anos com 43 (89,6\%) casos. O período de incubação médio foi de 27,6 dias, com variações de $3(6,2 \%)$ casos com menos de 15 dias e $4(8,3 \%)$ acima de 40 dias. Houve predomínio das lesões em membros superiores em 34 pacientes, seguindo-se face em 15 e pescoço em 10, outras localizações menos freqüentes ocorreram em tronco, orelha e membros inferiores. Em 24 (50\%) indivíduos havia duas lesões, em $8(16,7 \%)$ havia lesão única e somente $1(2,1 \%)$ enfermo apresentou mais de 10 lesões. Quanto ao aspecto clínico, as lesões ulcerosas foram as manifestações mais freqüentes, ocorrendo como forma exclusiva em 32 $(66,7 \%)$ pacientes e associadas a outros aspectos clínicos tais como impetigóides, papulosas, ectimatóides ou verrucosas, observadas em $11(30 \%)$ indivíduos. As leishmanides estavam presentes em $5(10,4 \%)$ casos e a linfangite foi detectada em 29 (60,4\%). Em 21 (43,8\%) pacientes havia concomitância com outras infecções de pele, sendo mais freqüentes as candidíases e as dermatofitoses, ocorrendo a associação de ambas em seis casos.

Em 43 (89,6\%) casos o diagnóstico foi confirmado, através do exame direto da escarificação da lesão. 0 exame direto foi negativo em cinco pacientes: destes, 3 $(6,2 \%)$ tiveram a IDRM positiva, $1(2,1 \%)$ apresentava exame histopatológico compatível com LTA e cultura positiva para Leishmania sp e 1 (2,1\%) enfermo teve diagnóstico clínico, sem confirmação laboratorial (Tabela 1), entretanto respondeu ao tratamento com pentamidina.

Os antimoniais foram inicialmente utilizados em $21(43,8 \%)$ doentes. Destes, $3(6 \%)$ doentes tiveram suas lesões cicatrizadas com uma série de antimoniais e $3(6 \%)$ com duas séries. Os outros $15(31,2 \%)$ pacientes utilizaram pentamidina como segunda linha de tratamento, sendo que $10(66,7 \%)$ receberam apenas uma série e $5(33,3 \%)$ utilizaram uma segunda série de pentamidina. Os efeitos colaterais relacionados aos antimoniais foram artralgia, astenia e febre.

A pentamidina foi utilizada como primeira linha em $27(56,2 \%)$ doentes, $15(31,2 \%)$ tiveram suas lesões cicatrizadas, $11(22,9 \%)$ utilizaram uma segunda série e $1(2,1 \%)$ utilizou três séries (Tabela 2$)$. Entre os pacientes que utilizaram pentamidina observou-se dor e formação de plastrão no local das injeções e sintomas de hipotensão, em um reduzido número de casos, imediatamente após a aplicação da medicação.

\section{DISCUSSÃO}

A LTA constitui importante problema de saúde pública no Estado do Amazonas, principalmente na cidade de Manaus, onde o êxodo rural foi propiciado por fatores tais como grandes enchentes do rio 
Tabela 1 - Leishmaniose tegumentar americana em militares simultaneamente expostos à infecçâo na Amazônia. Positividade dos métodos diagnósticos.

\begin{tabular}{lcc}
\hline & \multicolumn{2}{c}{ Número de pacientes } \\
\cline { 2 - 3 } Exames realizados & examinados & positivos \\
\hline Exame direto & 48 & 43 \\
IDRM & 3 & 3 \\
Histopatológico & 2 & 1 \\
\hline
\end{tabular}

Tabela 2 - Leishmaniose tegumentar americana em militares simultaneamente expostos à infecçâo na Amazônia. Demonstrativo da resposta terapêutica de acordo com a medicação e os esquemas de tratamento utilizados

\begin{tabular}{|c|c|c|c|c|c|}
\hline \multirow[t]{3}{*}{ Medicamento } & \multirow[t]{3}{*}{ Escolha } & \multirow[t]{3}{*}{ Série do medicamento } & \multicolumn{3}{|c|}{ Número de pacientes } \\
\hline & & & \multirow[t]{2}{*}{ tratados } & \multicolumn{2}{|c|}{ curados } \\
\hline & & & & $\mathrm{n}^{\circ}$ & $\%$ \\
\hline \multirow[t]{2}{*}{ Antimonial } & primeira linha & $1^{a *}$ & 21 & 3 & 6,2 \\
\hline & & $2^{\mathrm{a}}$ & 18 & 3 & 33,3 \\
\hline \multirow[t]{5}{*}{ Pentamidina } & primeira linha & $1^{a \star *}$ & 27 & 15 & 31,2 \\
\hline & & $2^{a}$ & 11 & 6 & 54,5 \\
\hline & & $3^{a}$ & 1 & 1 & 100,0 \\
\hline & segunda linha & $1^{\mathrm{a}}$ & 15 & 10 & 67,0 \\
\hline & & $2^{\mathrm{a}}$ & 5 & 5 & 100,0 \\
\hline
\end{tabular}

* cada série de antimoniais corresponde a 20 doses diárias da medicação

** cada série de pentamidina corresponde a três doses da medicação

Amazonas, associados à implantação da Zona Franca e posterior instalação do Distrito Industrial. A busca de trabalho e de melhores condições de vida têm ocasionado desmatamentos e assentamentos populacionais nas áreas periféricas de Manaus, fator importante para a elevada incidência da doença ${ }^{411}{ }^{17}$. A abertura de estradas e as conseqüentes ocupações para a agricultura é outro fator importante no aparecimento de novos casos de LTA.

Por último, salientamos a relativa freqüência da LTA em militares. Os treinamentos realizados no período de alta pluviosidade (novembro a maio) possibilitam aumento do número de indivíduos acometidos ${ }^{15}$. Os casos do presente estudo evidenciam que a ocorrência da LTA no município de Manaus continua sendo problema de grande importância em saúde pública, relacionado principalmente a dois fatores: em primeiro lugar, a sazonalidade, com maior ocorrência de casos no período chuvoso ${ }^{1014}$, e em segundo lugar, a invasão do homem aos habitats naturais dos transmissores ${ }^{14}$. Somente na FMT, de janeiro de 1997 a novembro de 2001, foram registrados 140 casos de LTA em militares (JAO Guerra: dados não publicados).

De acordo com a bibliografia consultada, a observação da LTA em militares foi documentada incialmente por Azulay \& Salgado ${ }^{3}$ que relataram a ocorrência de LTA em um grupo de militares na Amazônia. Posteriormente, Mattos et $\mathrm{al}^{14}$ descrevem os aspectos clínico-epidemiológicos da doença de um grupo de militares, que esteve em quatro áreas endêmicas diferentes, observando-se 27 (76,5\%) com IDRM positivo e $8(23,5 \%)$ com lesões de LTA, provavelmente contraídas em operações de selva em Manaus, pois a Leishmania isolada de todos os casos foi a Leishmania (V.) guyanensis.

Em relação aos aspectos terapêuticos observados, à semelhança dos resultados registrados na mesma região por Romero et $\mathrm{al}^{16}$, a resposta a apenas uma série de antimoniais foi baixa, principalmente quando, comparada ao grupo que utilizou pentamidina.

Brandão-Filho ${ }^{7}$ descreve, em um contingente de 545 militares que participaram de treinamentos em uma base militar da Zona da Mata em Pernambuco, a ocorrência de LTA IDRM positiva em 24,1\% deste grupo, onde 26 pessoas apresentaram quadro clínico de LTA. O vetor predominante foi a Lu choti, em um percentual de $89,9 \%$ entre os flebotomíneos identificados, o que sugere sua incriminação como principal vetor, embora a $L u$ wellcomei também tenha sido capturada na área.

Segundo Desjeux ${ }^{8}$, uma consciência permanente, baseada em educação em saúde continuada somada a uma estrita vigilância epidemiológica são cruciais para garantir a redução dos riscos. Assim sendo, as operações de treinamento militar na região devem ser planejadas para períodos em que a incidência da doença seja menor, a fim de que se minimizem o contacto com o vetor e evitem-se ou reduzam-se o número de casos.

\section{AGRADECIMENTOS}

Ao Dr. Gottfried Schmer, pela tradução do sumário para o inglês, a Dra. Alcidéa Rêgo Bentes e Souza e ao Dr. Marcus Luíz Barroso Barros pela revisão e sugestões do manuscrito. 


\section{REFERÊNCIAS BIBLIOGRÁFICAS}

1. Andrade SL. Leishmaniose Tegumentar Americana em área de ocupação recente na periferia da cidade de Manaus Amazonas Brasil. Dissertação de Mestrado. Fundação Oswaldo Cruz Rio de Janeiro, 1998.

2. Arias Junior J, Freitas RA. On the vectors of cutaneous leishmaniasis in the Central Amazon of Brazil. Acta Amazônica 7:507-527, 1977.

3. Azulay RD, Salgado U. Surto epidêmico de Leishmaniose Tegumentar observado em paraquedistas do exército na Amazônia. Medicina Cutânea Ibero Latino-americana. 4:34752, 1966.

4. Barret TV, Senra MS. Leishmaniasis in Manaus Brazil. Parasitology Today. 8:255-257, 1989

5. Barros MLB, Paes MG, Talhari S. Leishmaniose mucocutânea na Amazônia. Estudo dos casos diagnosticados em Manaus no período de 1976 a 1980. Anais Brasileiros de Dermatologia 57:152-54, 1982

6. Barros MLB, Paes MG, Toledo LM. Considerações sobre a produção da Leishmaniose Tegumentar Americana no Estado do Amazonas. In: Espaço e Doença: um olhar sobre o Amazonas. Editora FIOCRUZ, Rio de Janeiro. p. 105-113, 1998.

7. Brandão-Filho SP, Brito MEF, Martins CAP, Sommer IB, Valença HF, Almeida FA, Gomes J. Leishmaniose tegumentar americana em centro de treinamento militar localizado na Zona da Mata de Pernambuco. Revista da Sociedade Brasileira de Medicina Tropical 31:575-578, 1998.

8. Desjeux P. The increase in risk factors for leishmaniasis worldwide. Transactions of the Royal Society of Tropical Medicine and Hygiene 95:239-243, 2001.

9. Fundação Nacional de Saúde. Guia de Controle da Leishmaniose Tegumentar Americana. Ministério da Saúde. 3aㅡ edição Brasília, 1994.

10. Grimaldi Junior G, Tesh RB, McMahon-Pratt D. A review of geographic distribution and epidemiology of leishmaniasis in the New World American Journal of Tropical Medicine and Hygiene. 41 687-725, 1989.

11. Guerra JAO, Barros MLB, Guerra MVF, Talhari S, Paes MG Leishmaniose Tegumentar no município de Manaus - Aspectos epidemiológicos. In: Resumos do XXXIV Congresso da Sociedade Brasileira de Medicina Tropical, Manaus p.172, 1998.

12. Lainson $R$. The american leishmaniases: some observations on their ecology and epidemiology. Transactions of the Royal Society of Tropical Medicine and Hygiene 77:169-96, 1983.

13. Lainson R, Shaw JJ, Silveira FT, Souza AAA, Braga RR, Ishikawa EAl. The dermal Leishmaniasis of Brazil with special reference to the eco-epidemiology of the disease in the Amazonian. Memórias do Instituto Oswaldo Cruz 89:435-443, 1994.

14. Mattos MS, Grimaldi G, Cupolillo E, Pirmez C, Oliveira-Neto MP. Aspectos clínicos e epidemiológicos de um surto de Leishmaniose Tegumentar Americana (LTA) num grupo de militares do Rio de Janeiro. In: Resumos do XXXIV Congresso da Sociedade Brasileira de Medicina Tropical, Manaus p.129, 1998.

15. Paes MG. Estudo de quatro espécies de Lutzomyia França 1924 (Diptera Psychodidae) em área endêmica de Leishmaniose Tegumentar Americana na periferia de Manaus. Dissertação de Mestrado. Instituto Nacional de Pesquisas da Amazônia/ Fundação Universidade do Amazonas. Manaus, 1991.

16. Romero GAS, Guerra MVF, Paes MG, Macêdo VO. Comparison of Cutaneous Leishmaniasis due to Leishmania (Viannia) braziliensis and L. (V.) guyanensis in Brazil: Clinical findings and diagnostic approach. Clinical Infectious Diseases 32: 1304 1312, 2001.

17. Talhari S, Arias JR, Cunha MG, Naiff RD, Freitas R, Barret TV. Leishmaniose no Estado do Amazonas. Aspectos Clínicos Epidemiológicos e Terapêuticos. Anais Brasileiros de Dermatologia 6: 433-438, 1988. 